\title{
Regulated Water Deficits Improve Phytochemical Concentration in Lettuce
}

\author{
Myung-Min Oh \\ Department of Horticultural Science, Chung-Buk National University, Cheong-Ju, Korea \\ 361-763 \\ Edward E. Carey \\ K-State Horticulture Research and Extension Center, 35230 West 135th Street, Olathe, KS \\ 66061-9423 \\ C.B. Rajashekar ${ }^{1}$ \\ Division of Horticulture, Kansas State University, Manhattan, KS 66506
}

\begin{abstract}
AdDitional INDEX WORDs. antioxidants, Lactuca sativa, phenolic compounds, vitamins
ABSTRACT. In a growth chamber study, lettuce (Lactuca sativa) plants were used to evaluate the effects of water deficits on health-promoting phytochemicals with antioxidant properties. Lettuce plants were treated with water stress by withholding water once at 6 weeks after sowing for 2 days or multiple times at 4 weeks for 4 days, at 5 weeks for 3 days, and at 6 weeks for 2 days. Water stress increased the total phenolic concentration and antioxidant capacity in lettuce. Young seedlings, 7 days after germination, had the highest total phenolic concentration and antioxidant capacity, and also, younger plants were typically more responsive to water stress treatments in accumulating the antioxidants than older plants. Phenylalanine ammonia lyase and $\gamma$-tocopherol methyltransferase genes, involved in the biosynthesis of phenolic compounds and vitamin $\mathrm{E}$, respectively, were activated in response to water stress, although no activation of L-galactose dehydrogenase was detected. Lettuce plants subjected to multiple water stress treatments accumulated significant amounts of chicoric acid compared with the control plants. Although the increase in antioxidant activity in water stress-treated plants at harvest was not as great as in young seedlings, it was significantly higher than the control. One-time water stress treatment of lettuce at the time of harvest did not result in any adverse effect on plant growth. Thus, these results show that mild water stress in lettuce applied just before harvest can enhance its crop quality with regard to its phytochemical concentration without any significant adverse effect on its growth or yield.
\end{abstract}

In recent years, the potential importance of fruit and vegetables as health-promoting foods has grown in light of the increasing number of epidemiological studies suggesting that the daily intake of fruit and vegetables has significant health benefits in preventing many chronic and degenerative diseases (Arai et al., 2000; Berger, 2005; Block et al., 1992; Buring and Hennekens, 1997). This is because they are rich in numerous phytochemicals, including antioxidants (DellaPenna, 1999). Lettuce contains various health-promoting phytochemicals, including vitamins and phenolic compounds with antioxidant properties (Caldwell, 2003; Llorach et al., 2004; Nicolle et al., 2004). Among the major phenolic compounds in lettuce known to have health-promoting qualities are chlorogenic acid, caffeic acid, and chicoric acid (Graefe and Veit, 1999; Olthof et al., 2001).

In response to water stress, plants typically accumulate a wide range of antioxidants, including enzymatic antioxidants (peroxidases, superoxide dismutase, and catalase) and nonenzymatic antioxidants (phytochemicals) to quench the reactive oxidative species (ROS) induced by stress (Sofo et al., 2005). Water stress has been shown to induce a number of such phytochemicals, including $\alpha$-tocopherol, $\beta$-carotene, and flavonoids, in a wide range of plant species (Keles and Öncel, 2002;

Received for publication 28 Jan. 2010. Accepted for publication 6 Apr. 2010. This work was supported by the Kansas Agricultural Experiment Station and is a contribution (0-060-J) of the Kansas Agricultural Experiment Station.

${ }^{1}$ Corresponding author. E-mail: crajshe@ksu.edu.
Munné-Bosch et al., 2001; Tattini et al., 2004; Zobayed et al., 2007).

Because lettuce is the most commonly consumed fresh leafy vegetable, our focus in this study was to improve its phytochemical concentration using mild water stress at different stages of plant growth. We examined if regulated water stress could enhance the antioxidant capacity and phenolic concentration in lettuce. In addition, we determined if these changes are controlled through gene action by examining the activation of key genes involved in the biosynthesis of phenolic compounds and vitamin $\mathrm{C}$ and $\mathrm{E}$ in lettuce plants.

\section{Materials and Methods}

Plant material and growth conditions. Lettuce (cv. Baronet) seeds (Johnny's Selected Seeds, Winslow, MA) were germinated in pots $(8 \mathrm{~cm}$ long $\times 8 \mathrm{~cm}$ wide $\times 7 \mathrm{~cm}$ high $)$ containing a commercial growing medium (Metro-Mix 350; Sun Gro, Vancouver, BC, Canada). After germination, seedlings were thinned to retain a single seedling per pot. The growing medium was maintained at field capacity using a capillary subirrigation system. When the plants were 3 weeks old, they were fertilized weekly with a nutrient solution containing $20 \mathrm{~N}-4.4 \mathrm{P}-16.6 \mathrm{~K}$ at $250 \mathrm{mg} \cdot \mathrm{L}^{-1} \mathrm{~N}$. These green leaf-type lettuce plants (36 plants per treatment) were grown with a 12 -h photoperiod at $22 / 18{ }^{\circ} \mathrm{C}$ (day/night) and a photosynthetic photon flux $(P P F)$ of $250 \mu \mathrm{mol} \cdot \mathrm{m}^{-2} \cdot \mathrm{s}^{-1}$ in two growth chambers. 
WATER Stress Treatment. Water stress treatments were initiated when seedlings were 4-week-old plants. Water stress was induced in plants by withholding watering. To make sure that plants were treated with mild water stress, they were rewatered as the plants began to lose turgor. Typically, withholding water for $4 \mathrm{~d}$ at 4 weeks of seeding resulted in a leaf water potential of about $-1.5 \mathrm{MPa}$. Lettuce plants were treated with water stress once at 6 weeks (before harvest) for $2 \mathrm{~d}$ or multiple times at 4 weeks for $4 \mathrm{~d}$, at 5 weeks for $3 \mathrm{~d}$, and at 6 weeks for $2 \mathrm{~d}$.

LEAF WATER POTENTIAL. Leaf water potential was measured daily during the water stress treatment by a thermocouple psychrometer (Tru Psi; Decagon Devices, Pullman, WA). Three leaf disks (1.2 cm in diameter) from fully expanded leaves of 4-week-old plants were cut with a cork borer and were immediately transferred to small sealed stainless steel chambers. Each treatment had three replications. The samples were equilibrated at $25^{\circ} \mathrm{C}$ for $50 \mathrm{~min}$ before the measurement. Water potential of the leaf disks was measured with a cooling time of $15 \mathrm{~s}$.

LEAF CHLOROPHYLL FLUORESCENCE. To evaluate the photosynthetic efficiency of lettuce, chlorophyll fluorescence was measured on the adaxial side of a fully expanded leaf with a chlorophyll fluorometer (PEA; Hansatech Instruments, Norfolk, UK) during the water stress treatment. The fully expanded leaves from the middle of the canopy of 4-week-old plants were selected for the measurement. Fv/Fm ratio (potential quantum yield) was measured during the day using the saturation pulse method after 30 min of dark adaptation. Three plants were used for each measurement.

Total Phenolic Concentration. Lettuce leaves were collected every 3 or $4 \mathrm{~d}$ from 1 to 6 -week-old plants to monitor the changes in total phenolic concentration. Total phenolic concentration of lettuce leaves was analyzed by a modified FolinCiocalteu reagent method (Ainsworth and Gillespie, 2007) as outlined elsewhere (Oh et al., 2009). Three lettuce plants per treatment were used for the analysis. Collected samples were stored at $-20{ }^{\circ} \mathrm{C}$ until use.

Antioxidant CAPACITY. Leaf samples for this assay were collected at the same time as for the determination of total phenolic concentration. Antioxidant capacity of lettuce leaves was monitored by a modified 2,2'-azino-bis(3-ethylbenzthiazoline-6sulphonic acid) (ABTS) method (Awika et al., 2003; Miller and Rice-Evans, 1996; Pennycooke et al., 2005) as described previously by $\mathrm{Oh}$ et al. (2009).

RNA GEL BLOT ANALYSIS. RNA gel blot analysis was conducted to determine the activation of key genes involved in the biosynthesis of phytochemicals. The activation of genes for L-galactose dehydrogenase (L-GalDH), $\gamma$-tocopherol methyltransferase $(\gamma$-TMT), and phenylalanine ammonia lyase (PAL) involved in the biosynthesis of vitamin $\mathrm{C}$, vitamin $\mathrm{E}$, and phenolic compounds, respectively (Bergmüller et al., 2003; Diallinas and Kanellis, 1994; Gatzek et al., 2002), was determined. The specific probes for L-GalDH (497 bp, accession no. AJ417563) with primers 5'-AACTTCTTCGACACCTCCCC$3^{\prime}$ and $5^{\prime}$-TCATCCCAACCAACACCGAC-3', for $\gamma$-TMT (244 bp, accession no. AF104220) with primers 5'-CATAGAA ATCTATCTGCGGG-3' and 5' - CACTACGAAGCAGAGAC ACA-3', and for PAL (753 bp, accession no. AF299330) with primers 5' -GCTTACAGTTTCTCAGGTGG-3' and 5' -TGAT GCTTCAATTTGTGTGT-3' were amplified using genomic DNA from lettuce plants with the primer pairs designed by a primer design program (Primer3; Whitehead Institute, Cambridge, MA). Amplification of probes was performed by a Peltier thermal cycler (PTC-100; Bio-Rad, Hercules, CA) after initial denaturation at $94{ }^{\circ} \mathrm{C}$ for $5 \mathrm{~min}$ by 35 cycles of $1 \mathrm{~min}$ at $94{ }^{\circ} \mathrm{C}, 1 \mathrm{~min}$ at $51{ }^{\circ} \mathrm{C}(\gamma$-TMT and PAL $)$ or $54{ }^{\circ} \mathrm{C}$ (L-GalDH), and $1 \mathrm{~min}$ at $72^{\circ} \mathrm{C}$, followed by $5 \mathrm{~min}$ at $72^{\circ} \mathrm{C}$. The fragment size of each probe was checked by electrophoresis on $1.2 \%(\mathrm{w} / \mathrm{v})$ agarose gels in $1 \times$ Tris-acetate-EDTA buffer. The fragments were stained with ethidium bromide and checked by an ultraviolet transilluminator (FisherBiotech, Fairlawn, NJ). The probes were stored at $-20{ }^{\circ} \mathrm{C}$ until use.

The samples were collected to check the transcript levels of target genes at 6 weeks of planting. Total RNA was extracted from lettuce leaves using a modified cetyltrimethylammonium bromide (CTAB) extraction method as described by Chang et al. (1993). Total RNA $(20 \mu \mathrm{g})$ was subjected to electrophoresis on $1 \%(\mathrm{w} / \mathrm{v})$ agarose gels with $10 \%(\mathrm{v} / \mathrm{v})$ formaldehyde. The RNA gel blot was performed as described elsewhere (Oh et al., 2009).

High-PERformance LiQuid Chromatography (HPLC) ANALYSES. Six-week-old lettuce plants were used for HPLC analyses. Fully developed leaves from three plants per treatment were collected for the analysis of each phytochemical component. Collected samples were stored at $-20{ }^{\circ} \mathrm{C}$ until use.

Phenolic compounds. The extraction of phenolic compounds from lettuce leaves was carried out as described by Nicolle et al. (2004) with minor modifications. The HPLC analyses were conducted as described by Oh et al. (2009), and peaks from the sample extract were identified and quantified at $330 \mathrm{~nm}$ by comparing with standard compounds, namely, chlorogenic acid (Sigma-Aldrich, St. Louis), caffeic acid (Sigma-Aldrich), and chicoric acid (Indofine Chemical, Hillsborough, NJ).

AscorbIC ACID. Ascorbic acid was extracted from $1 \mathrm{~g}$ of lettuce leaves frozen in liquid nitrogen as described by Wimalasiri and Wills (1983), with some modifications. The samples were ground in a mortar with a pestle and then extracted with $10 \mathrm{~mL}$ of $3 \%$ metaphosphoric acid $\left(\mathrm{HPO}_{3}\right)$ (Riedel-de Haën, Seelze, Germany). Sample mixed with metaphosphoric acid solution was filtered through a filter paper (No. 1; Whatman, Maidstone, UK) and subsequently with a $0.45-\mu \mathrm{m}$ filter ascrodisc (Millex; Millipore, Bedford, MA) under ice. The extract $(5 \mu \mathrm{L})$ was immediately injected into a HPLC system equipped with a column (Discovery BIO Wide Bore C-18, $15 \mathrm{~cm} \times 4.6 \mathrm{~mm}, 5 \mu \mathrm{m}$; Supelco, Bellefonte, PA) and a guard column (Discovery BIO Wide Bore C-18, $2 \mathrm{~cm} \times$ $4 \mathrm{~mm}, 5 \mu \mathrm{m}$; Supelco) at $25^{\circ} \mathrm{C}$. The mobile phase was $10 \mathrm{~mm}$ ammonium dihydrogen phosphate $\left[\left(\mathrm{NH}_{4}\right) \mathrm{H}_{2} \mathrm{PO}_{4}, \mathrm{pH} 4\right]$ at a flow rate of $1 \mathrm{~mL} \cdot \mathrm{min}^{-1}$. Ascorbic acid was detected at $255 \mathrm{~nm}$ and was quantified by the ascorbic acid standards (SigmaAldrich).

$\alpha$-Tocopherol. The extraction of $\alpha$-tocopherol was performed following the method of Kim et al. (2007), with minor modifications. Homogenized leaf sample (1 g) was extracted with $3 \mathrm{~mL}$ of hexane (Chromasolv plus; Sigma-Aldrich) containing $0.1 \%$ butylated hydroxyl toluene (BHT; SigmaAldrich) and the organic phase was collected. The remaining pellet was extracted again with $3 \mathrm{~mL}$ of hexane containing $0.1 \%$ BHT. The combined organic phase was adjusted to $5 \mathrm{~mL}$ and was filtered through a $0.45-\mu \mathrm{m}$ filter ascrodisc (Millex; Millipore) in the dark at $4{ }^{\circ} \mathrm{C}$. The extract was evaporated to dryness on ice under nitrogen and was then resuspended in $1 \mathrm{~mL}$ of 
methanol. A $20-\mu \mathrm{L}$ extract was injected into a HPLC equipped with a column (Discovery BIO Wide Bore C-18, $15 \mathrm{~cm} \times$ $4.6 \mathrm{~mm}, 5 \mu \mathrm{m}$; Supelco) and a guard column (Discovery BIO Wide Bore C-18, $2 \mathrm{~cm} \times 4 \mathrm{~mm}, 5 \mu \mathrm{m}$; Supelco). A flow rate of $1.8 \mathrm{~mL} \cdot \mathrm{min}^{-1}$ was used with the mobile phase consisting of $97 \%$ methanol and $3 \%$ water. The wavelengths were set at $295 \mathrm{~nm}$ for excitation and $340 \mathrm{~nm}$ for emission to determine $\alpha$-tocopherol. The samples were identified and quantified using the standard $\alpha$-tocopherol (Sigma-Aldrich).

Plant growth Characteristics. Plant growth characteristics were measured at the end of the experiment, 6 weeks after planting. Dry and fresh weights of roots and shoots, total leaf area, and total root length, volume, and surface area were measured using four plants per each treatment. After measuring fresh weights of roots and shoots, the samples were dried at 70 ${ }^{\circ} \mathrm{C}$ in an oven for $3 \mathrm{~d}$ to measure their dry weights. Total leaf area was measured by a leaf area meter (LI-3100C; LI-COR, Lincoln, NE). Total root length, and volume and surface area were measured using a root scanning system (WinRHIZO; Regent Instruments, Quebec, QC, Canada). Roots were washed thoroughly in flowing tap water and were stained with methyl blue solution $\left(5 \mathrm{~g} \cdot \mathrm{L}^{-1}\right)$ for $5 \mathrm{~min}$. Stained roots were washed again with tap water and were carefully spread on a transparent plastic support for scanning.

STATistical analysis. Analysis of variance (ANOVA) was performed by SAS (version 9.0; SAS Institute, Cary, NC). The experiment was conducted using a randomized complete block design with three replications. Means were compared using Duncan's multiple range test.

\section{Results}

The highest concentration of total phenolic compounds was observed in young lettuce seedlings $7 \mathrm{~d}$ following the germination; however, it rapidly decreased by more than $60 \%$, in just about $14 \mathrm{~d}$. Following this rapid drop, the decrease in the overall total phenolic concentration was rather gradual until harvest (Fig. 1). The changes in antioxidant capacity of lettuce plants were roughly reflective of the changes in the total phenolic concentration. When 4-week-old lettuce plants were subjected to water stress by withholding water for up to $4 \mathrm{~d}$, the leaf water potential initially decreased gradually, followed by a sharp decline to about $-1.5 \mathrm{MPa}$ on the fourth day, as reflected by the loss of leaf turgor. Rewatering the plants following the stress period resulted in rapid recovery of turgor and leaf water potential (Fig. 2). During water stress, there was a gradual decrease in the leaf fluorescence $(\mathrm{Fv} / \mathrm{Fm}) . \mathrm{Fv} / \mathrm{Fm}$ appeared to be more sensitive to mild water stress, but increased when plants were subjected to more severe water stress at $4 \mathrm{~d}$ of water deficit, as indicated by leaf water potential.

When 4-week-old lettuce plants were subjected to water stress multiple times (three times), the total phenolic concentration in plants increased each time, with the highest increase in younger plants at 4 weeks (Fig. 3). It is interesting to note that the rewatering of plants following the stress period resulted in a decrease in their total phenolic concentration, suggesting that the levels of total phenolics (expressed on dry weight basis) are quite sensitive to the water status of plants. The total phenolic accumulations were less responsive to water stress as they aged; however, it is important to note that water stress just before harvest produced significant increase in total phenolic compounds and antioxidant capacity compared with the control.

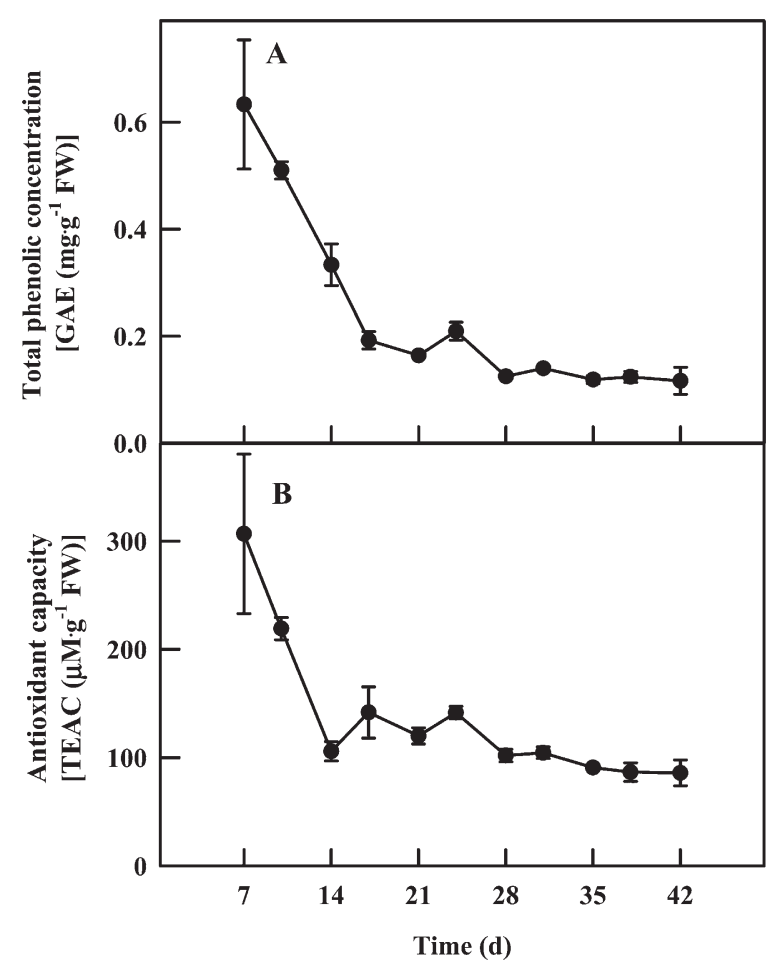

Fig. 1. Changes in (A) total phenolic concentration [gallic acid equivalent (GAE)] and (B) antioxidant capacity [trolox equivalent antioxidant capacity (TEAC)] in lettuce leaves with plant age. Lettuce plants were grown at 22 / $18^{\circ} \mathrm{C}$ (day/night), $250 \mu \mathrm{mol} \cdot \mathrm{m}^{-2} \cdot \mathrm{s}^{-1} P P F$, and a $12-\mathrm{h}$ photoperiod in a growth chamber. The values are the means of three lettuce plants. Bars represent SE.

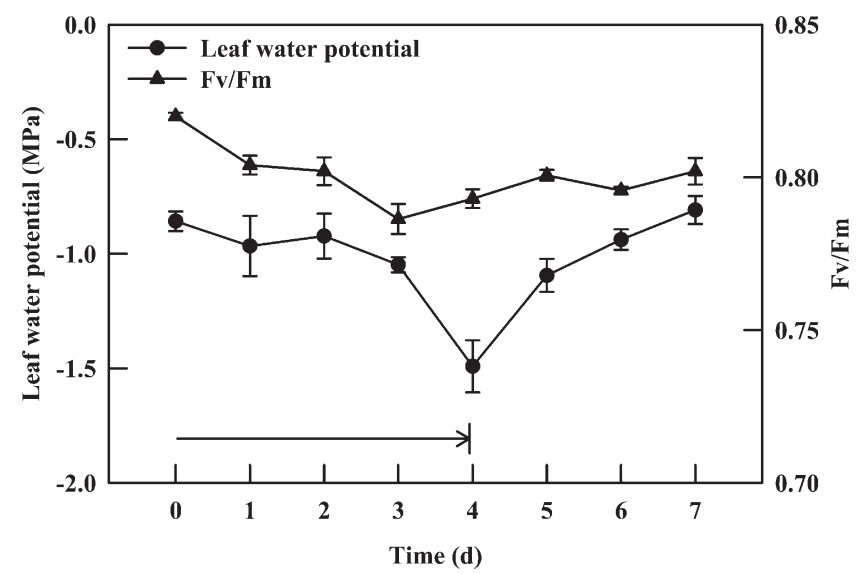

Fig. 2. Leaf water potential and Fv/Fm in 4-week-old lettuce plants subjected to water withholding for $4 \mathrm{~d}$. Leaf water potential and Fv/Fm were measured during the water stress period. The period of water withholding is indicated by the arrow and was followed by rewatering. Three lettuce plants were used for each measurement. Bars represent SE.

Also, the changes in the antioxidant capacity of water-stressed plants paralleled those in the total phenolic compounds, although the antioxidant capacity of the control plants was somewhat variable. It should be noted that at the time of harvest, there was no significant difference in total phenolic concentration and antioxidant capacity between plants treated with water stress one time and multiple times. However, these characteristics were significantly higher in water-stressed 


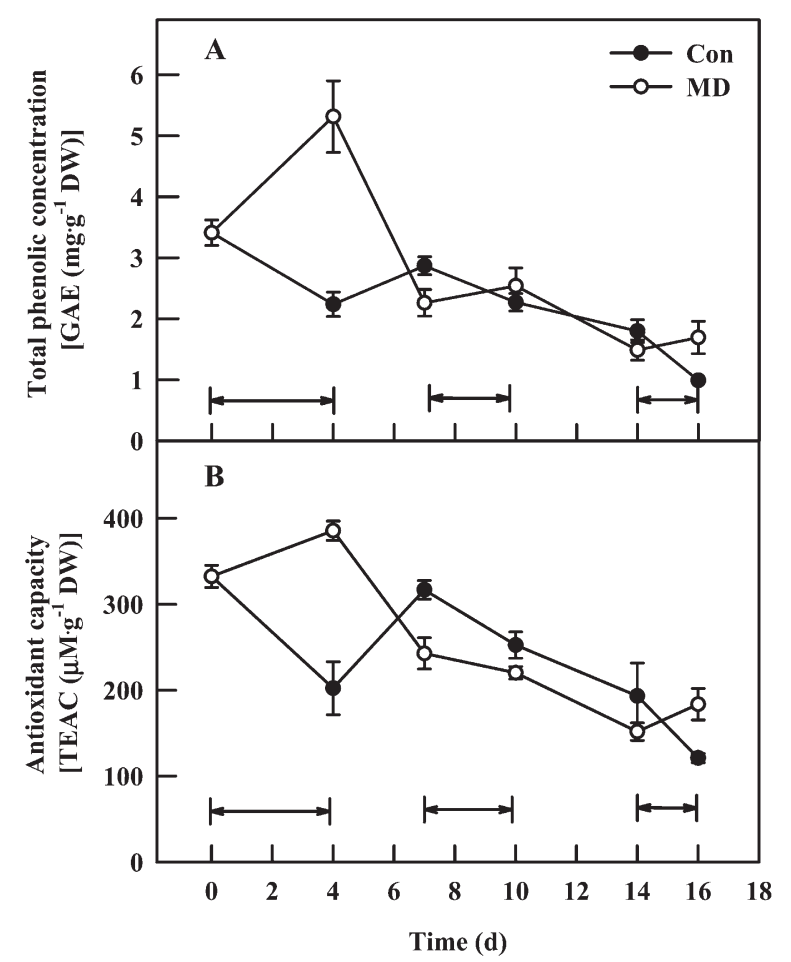

Fig. 3. (A) Total phenolic concentration [gallic acid equivalent (GAE)] and (B) antioxidant capacity [trolox equivalent antioxidant capacity (TEAC)] of lettuce leaves subjected to multiple water stress treatments. Lettuce plants were subjected to water stress by withholding water at 4 weeks for $4 \mathrm{~d}$, at 5 weeks for $3 \mathrm{~d}$, and at 6 weeks for $2 \mathrm{~d}$ after planting. Con and MD represent control and multiple water stress treatments, respectively. Control represents well-watered plants. Three lettuce plants were used per treatment. Bars represent SE. Arrows indicate drought stress periods.

plants, regardless of whether they were subjected to one time or multiple times, than in the control (unstressed) plants (Fig. 4).

To ascertain if the stress-induced accumulation of antioxidants is as a result of gene activation, expressions of various key genes, including PAL, $\gamma$-TMT, and L-GalDH, involved in the biosynthesis of antioxidants were measured. Water stress imposed, one time or multiple times, resulted in higher accumulation of PAL and $\gamma$-TMT transcripts in lettuce at the time of harvest, while the expression of L-GalDH was unaffected by the stress treatments (Fig. 5).

Chicoric acid, one of common phenolic acids in lettuce, accumulated in response to multiple exposures to water stress treatments, but not at significant levels to a single exposure to water stress (Fig. 6). In addition, no significant increase in caffeic acid or chlorogenic acid was noted in response to water stress. Similarly, no significant accumulation of ascorbic acid or $\alpha$-tocopherol in response to water stress was observed (Fig. 7).

With regard to the effect of water stress on plant growth in lettuce, no adverse affect on shoot or root growth was observed in response to a one-time exposure to water stress before harvest. There were no significant differences in biomass accumulation, leaf area, or root growth (root length, volume, and surface area) between the water-stressed plants and the control plants (Table 1). However, exposure of plants to multiple water stress treatments adversely affected plant growth in lettuce. Most of the shoot growth parameters such as fresh weight and leaf area declined slightly, except dry

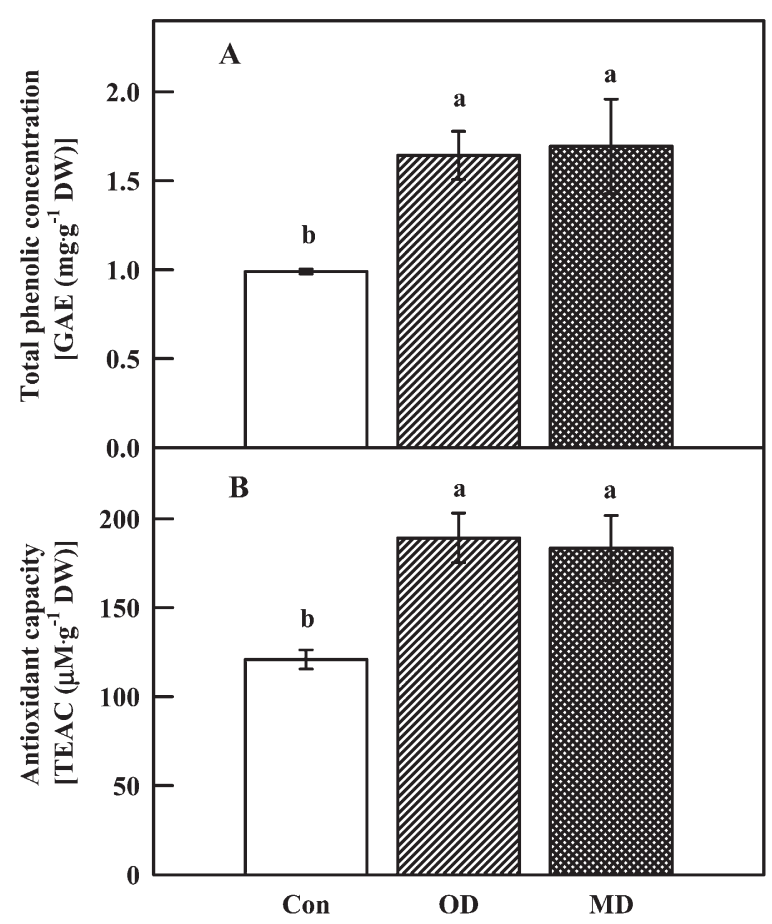

Fig. 4. (A) Total phenolic concentration [gallic acid equivalent (GAE)] and (B) antioxidant capacity [trolox equivalent antioxidant capacity (TEAC)] of lettuce leaves in plants subjected to a one-time or multiple water stress treatments. Lettuce plants were harvested at 6 weeks after planting. Con, OD, and MD represent control, one-time water stress (at 6 weeks for $2 \mathrm{~d}$ ), and multiple water stress treatments (at 4 weeks for $4 \mathrm{~d}$, at 5 weeks for $3 \mathrm{~d}$, and at 6 weeks for $2 \mathrm{~d}$ ), respectively. Control represents well-watered plants. Three lettuce plants were used per treatment. Bars represent SE. Significant differences between treatments via Duncan's multiple range test at $P \leq$ 0.05 are indicated by different letters.

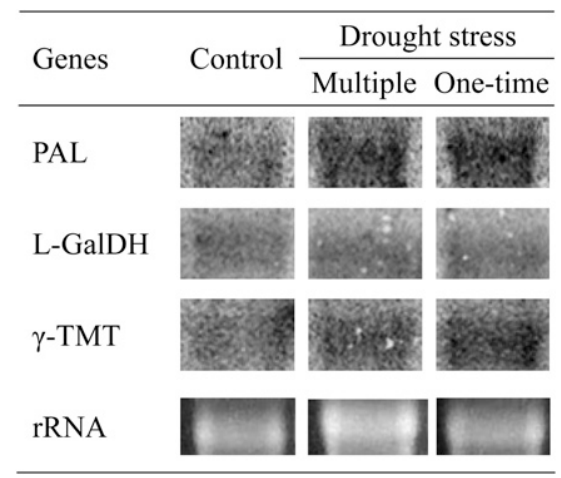

Fig. 5. The gene expression of phenylalanine ammonia lyase (PAL), Lgalactose dehydrogenase (L-GalDH), and $\gamma$-tocopherol methyltransferase $(\gamma$-TMT) in lettuce plants subjected to a one-time or multiple water stress treatments. Plants were harvested at 6 weeks after planting. Lettuce plants were subjected to one-time water stress (at 6 weeks for $2 \mathrm{~d}$ ) or multiple water stress treatments (at 4 weeks for $4 \mathrm{~d}$, at 5 weeks for $3 \mathrm{~d}$, and at 6 weeks for $2 \mathrm{~d}$ ). Control represents transcript levels of lettuce plants grown without water stress in a growth chamber. rRNA was used as a loading standard.

weight, in response to multiple stress events. Interestingly, root growth was negatively correlated with shoot growth. Plants treated with multiple stress treatments showed better root growth than the control or plants subjected to a single exposure to water stress treatment. Indeed, multiple stress events induced 


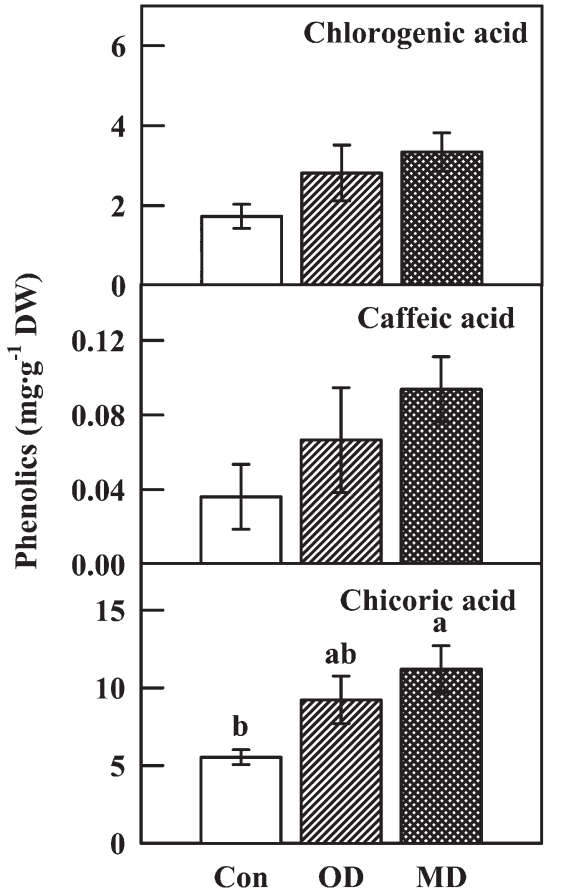

Fig. 6. Concentrations of individual phenolic compounds in lettuce plants subjected to a one-time or multiple drought stress treatments. Lettuce plants were harvested at 6 weeks after planting. Con, OD, and MD represent control, one-time water stress treatments (at 6 weeks for $2 \mathrm{~d}$ ), and multiple drought stress (at 4 weeks for $4 \mathrm{~d}$, at 5 weeks for $3 \mathrm{~d}$, and at 6 weeks for $2 \mathrm{~d}$ ), respectively. Control represents well-watered plants. Three lettuce plants were used per treatment. Bars represent SE. Significant differences between treatments via Duncan's multiple range tests at $P \leq 0.05$ are indicated by different letters.

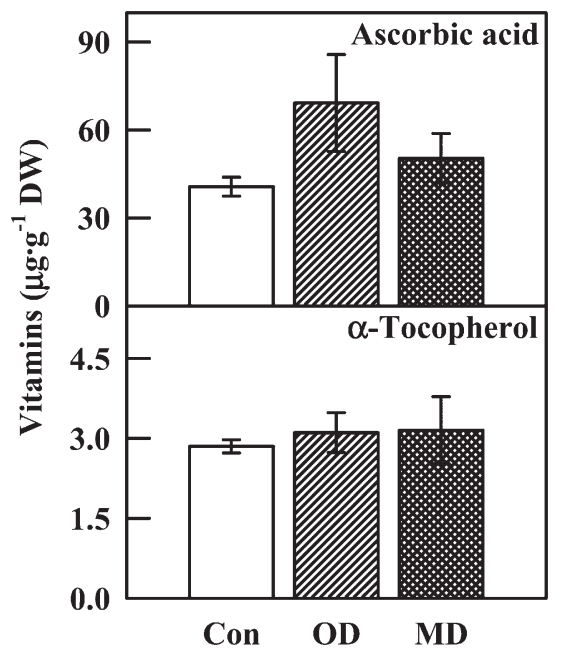

Fig. 7. Concentrations of ascorbic acid and $\alpha$-tocopherol in lettuce plants subjected to a one-time or multiple water stress treatments. Lettuce plants were harvested at 6 weeks after planting. Con, OD, and MD represent control, one-time water stress (at 6 weeks for $2 \mathrm{~d}$ ), and multiple water stress treatments (at 4 weeks for $4 \mathrm{~d}$, at 5 weeks for $3 \mathrm{~d}$, and at 6 weeks for $2 \mathrm{~d}$ ), respectively. Control represents well-watered plants. Three lettuce plants were used per treatment. Bars represent SE.

a significantly bigger root system characterized by higher root biomass accumulation, root length, volume, and surface area than in the control plants or in plants subjected to a single water stress treatment.

\section{Discussion}

Young lettuce seedlings were rich in phenolic compounds and antioxidants. However, total phenolic compounds and antioxidant capacity declined rapidly and reached their lowest level at the time of harvest. Germinating seeds (sprouts) and young seedlings have long been known for their health-promoting benefits (French et al., 1944). For example, compared with mature plants, sprouts of radish (Raphanus sativus), broccoli (Brassica oleracea var. italica), cauliflower (B. oleracea var. botrytis), and legumes are known to be rich in phytochemicals and thus to have a strong protective function against many types of degenerative diseases (Barillari et al., 2006; Fahey et al., 1997; Gill et al., 2004; Oh and Rajashekar, 2009). Typically, germinating seeds and young seedlings shift to secondary metabolism because of carbon limitation in germinating seeds, and the accumulation of secondary metabolites, especially antioxidants, may aid young seedlings against biotic and abiotic threats (Barton, 2007; Bryant et al., 1992; Clarke and Davison, 2004; Harper, 1977). In this study, as antioxidants declined with plant age to their lowest level at the time of harvest, the quality of lettuce in relation to its health-promoting value was also low compared with young seedlings.

However, when lettuce plants were exposed to mild water stress, there was a significant increase in their total phenolic concentration and antioxidant capacity. This result was consistent with our previous results from studies on lettuce and several edible sprouts exposed to other abiotic stresses such as heat shock, chilling, or high light (Oh et al., 2009; Oh and Rajashekar, 2009). Furthermore, numerous studies have shown that drought stress can induce a wide range of antioxidants in a number of plant species (Bray, 2002; Keles and Öncel, 2002; Munné-Bosch et al., 2001; Sofo et al., 2005). Our results showed that plant response to water stress in lettuce was very much dependent on plant age. Younger seedlings (4 weeks old) exposed to water stress increased their phenolic content and antioxidant capacity to a larger extent. In response to water stress, the increase in total phenolic concentration was about four times higher when plants were 4 weeks old compared with that in older plants. Thus, the results suggest that young lettuce plants are not only richer in phenolic compounds and higher in antioxidant capacity, but also are more responsive to mild water stress than older plants. Furthermore, the accumulation of phenolic compounds in lettuce plants appears to be sensitive to the plants' water status. This is demonstrated by the increase in phenolic compounds during water stress and the decrease in response to rewatering of plants. The results also suggest that the exposure of plants to water stress to multiple times did not have a cumulative or elevated response compared with a single exposure to water stress. The improvement of phytochemical concentration of lettuce plants treated with water stress once at 6 weeks before harvest or treated multiple times was identical. It should also be noted that although their response at the time of harvest ( 6 weeks) was much smaller than that in younger plants, the increase in phytochemicals was still quite significant.

In response to water stress, an increased activation of PAL was observed in lettuce. This response was consistent with the higher accumulation of phenolic compounds in lettuce. Expression of PAL in response to water stress is noteworthy because it is the gateway enzyme in the phenylpropanoid pathway that yields a wide range of phenolic compounds (Diallinas and Kanellis, 1994; Liu et al., 2006). Similarly, a higher transcript 
Table 1. Plant growth characteristics of lettuce subjected to water stress one time before harvest (OD) or multiple times (MD). Lettuce plants were harvested at 6 weeks after planting. Lettuce plants were subjected to a one-time water stress (at 6 weeks for 2 d) or multiple water stress treatments (at 4 weeks for $4 \mathrm{~d}$, at 5 weeks for $3 \mathrm{~d}$, and at 6 weeks for $2 \mathrm{~d}$ ). Control represents well-watered lettuce plants grown in a growth chamber. The values are the means of four lettuce plants per treatment.

\begin{tabular}{|c|c|c|c|c|c|c|c|c|}
\hline \multirow[b]{2}{*}{ Treatment } & \multicolumn{2}{|c|}{ Fresh wt (g) } & \multicolumn{2}{|c|}{ Dry wt (g) } & \multirow{2}{*}{$\begin{array}{l}\text { Total leaf } \\
\text { area }\left(\mathrm{cm}^{2}\right)\end{array}$} & \multirow{2}{*}{$\begin{array}{l}\text { Total root } \\
\text { length }(\mathrm{cm})\end{array}$} & \multirow{2}{*}{$\begin{array}{c}\text { Total root } \\
\text { surface }\left(\mathrm{cm}^{2}\right)\end{array}$} & \multirow{2}{*}{$\begin{array}{c}\text { Total root } \\
\text { volume }\left(\mathrm{cm}^{3}\right)\end{array}$} \\
\hline & Shoot & Root & Shoot & Root & & & & \\
\hline Control & $44.37 \mathrm{a}^{\mathrm{z}}$ & $5.19 \mathrm{~b}$ & 2.15 & $0.20 \mathrm{~b}$ & $882.5 \mathrm{a}$ & $4116.5 b$ & $540.3 \mathrm{~b}$ & $5.65 \mathrm{~b}$ \\
\hline OD & $41.64 \mathrm{a}$ & $5.17 \mathrm{~b}$ & 2.09 & $0.20 \mathrm{~b}$ & $813.5 \mathrm{a}$ & $3921.0 \mathrm{~b}$ & $538.5 \mathrm{~b}$ & $5.89 \mathrm{~b}$ \\
\hline
\end{tabular}

Significance

$*$

NS

${ }^{\mathrm{z}}$ Mean separation within columns by Duncan's multiple range test. Values in a column followed by the same letters are not significantly different. * Significant at $P=0.05$ via ANOVA.

NS $=$ Not significant.

level of $\gamma$-TMT was noted in response to water stress. However, no significant accumulation of $\alpha$-tocopherol was detected in the leaves. This clearly suggests that mild water stress can activate key genes involved in the biosynthesis of phytochemicals in lettuce, although the accumulation of the phytochemicals did not always occur, as in the case of $\alpha$-tocopherol. In fact, other environmental stresses involving chilling, heat, and high light have been shown to activate genes involved in the biosynthesis of some common antioxidants in lettuce (Oh et al., 2009). Thus, it is likely that phenolic accumulation in response to drought stress in lettuce is as a result of genetic control rather than chemical or physiological modulation of biosynthetic pathways.

Analyses of individual phenolic compounds indicate that the dominant phenolic compound in lettuce, chicoric acid, accumulates in response to water stress. In addition, levels of other phenolic compounds such as chlorogenic acid, caffeic acid, and vitamins such as ascorbic acid and $\alpha$-tocopherol in lettuce leaves did not change significantly. This suggests that in addition to chicoric acid, other antioxidants may contribute to the stress-induced increase in the total phenolic compounds and the antioxidant capacity in lettuce.

While plant stresses including water stress are generally considered to produce adverse effects on growth and development, it is important to assess their impact on biomass accumulation and crop yield (Boyer, 1982). Therefore, the stress treatments aimed at improving the antioxidant levels in plants need to be mild enough not to adversely affect plant growth. In this study, the exposure of lettuce plants to mild water stress once just before harvest produced a significant increase in phytochemicals without any adverse effect on plant growth. However, plant exposure to water stress multiple times, though equally as good in improving the antioxidant quality, produced a negative effect on shoot growth of lettuce.

\section{Conclusion}

Our results show that water stress imposed once before harvest was as good as that imposed multiple times during the plant growth with regard to enhancing the phytochemical concentration in lettuce. However, exposure of plants to multiple water stress resulted in a reduction in shoot growth, while a single exposure before harvest did not have any adverse effect on the growth of lettuce plants. Therefore, mild water stress before harvest would be of great value in improving the health-promoting attributes in lettuce. These results suggest the potential use of regulated water deficits as a crop management approach in improving the quality of lettuce crop by enhancing its nutritive and health-promoting value.

\section{Literature Cited}

Ainsworth, E.A. and K.M. Gillespie. 2007. Estimation of total phenolic content and other oxidation substrates in plant tissues using Folin-Ciocalteu reagent. Nat. Protoc. 2:875-877.

Arai, Y., S. Watanabe, M. Kimira, K. Shimoi, R. Mochizuki, and N. Kinae. 2000. Dietary intakes of flavonols, flavones and isoflavones by Japanese women and the inverse correlation between quercetin intake and plasma LDL cholesterol concentration. J. Nutr. 130:22432250.

Awika, J.M., L.W. Rooney, X. Wu, R.L. Prior, and L. CisnerosZevallos. 2003. Screening methods to measure antioxidant activity of sorghum (Sorghum bicolor) and sorghum products. J. Agr. Food Chem. 51:6657-6662.

Barillari, J., R. Cervellati, S. Costa, M.C. Guerra, E. Speroni, A. Utan, and R. Iori. 2006. Antioxidant and choleretic properties of Raphanus sativus L. sprout (kaiware daikon) extract. J. Agr. Food Chem. 54: 9773-9778.

Barton, K.E. 2007. Early ontogenetic patterns in chemical defense in Plantago (Plantaginaceae): Genetic variation and trade-offs. Amer. J. Bot. 94:56-66.

Berger, M.M. 2005. Can oxidative damage be treated nutritionally? Clin. Nutr. 24:172-183.

Bergmüller, E., S. Porfirova, and P. Dörmann. 2003. Characterization of an Arabidopsis mutant deficient in $\gamma$-tocopherol methyltransferase. Plant Mol. Biol. 52:1181-1190.

Block, G., B. Patterson, and A. Subar. 1992. Fruit, vegetables, and cancer prevention: A review of the epidemiologic evidence. Nutr. Cancer 18:1-29.

Boyer, J.S. 1982. Plant productivity and environment. Science 218:443-448.

Bray, E.A. 2002. Classification of genes differentially expressed during water-deficit stress in Arabidopsis thaliana: An analysis using microarray and differential expression data. Ann. Bot. (Lond.) 89:803-811.

Bryant, J.P., P.B. Reichardt, T.P. Clausen, F.D. Provenze, and P.J. Kuropat. 1992. Woody plant-mammal interactions, p. 344-371. In: G.A. Rosenthal and M.R. Berenbaum (eds.). Herbivores: Their interactions with secondary plant metabolites. Vol. 2, 2nd ed. Academic Press, San Diego.

Buring, J.E. and C.H. Hennekens. 1997. Antioxidant vitamins and cardiovascular disease. Nutr. Rev. 55:S53-S60.

Caldwell, C.R. 2003. Alkylperoxyl radical scavenging activity of red leaf lettuce (Lactuca sativa L.) phenolics. J. Agr. Food Chem. 51:4589-4595.

Chang, S.J., J. Puryear, and J. Cairney. 1993. A simple and efficient method for isolating RNA from pine trees. Plant Mol. Biol. Rpt. 11:113-116. 
Clarke, P.J. and E.A. Davison. 2004. Emergence and survival of herbaceous seedlings in temperate grassy woodlands: Recruitment limitations and regeneration niche. Austral Ecol. 29:320331.

DellaPenna, D. 1999. Nutritional genomics: Manipulating plant micronutrients to improve human health. Science 285:375-379.

Diallinas, G. and A.K. Kanellis. 1994. A phenylalanine ammonia-lyase gene from melon fruit: cDNA cloning, sequence and expression in response to development and wounding. Plant Mol. Biol. 26:473479.

Fahey, J.W., Y. Zhang, and P. Talalay. 1997. Broccoli sprouts: An exceptionally rich source of inducers of enzymes that protect against chemical carcinogens. Proc. Natl. Acad. Sci. USA 94:1036710372.

French, C.E., G.H. Berryman, J.T. Goorley, H.A. Harper, D.M. Harkness, and E.J. Hacker. 1944. The production of vitamins in germinated peas, soybeans, and other beans. J. Nutr. 28:63-70.

Gatzek, S., G.L. Wheeler, and N. Smirnoff. 2002. Antisense suppression of L-galactose dehydrogenase in Arabidopsis thaliana provides evidence for its role in ascorbate synthesis and reveals lights modulated L-galactose synthesis. Plant J. 30:541-553.

Gill, C.I.R., S. Haldar, S. Porter, S. Matthews, S. Sullivan, J. Coulter, H. McGlynn, and I. Rowland. 2004. The effect of cruciferous and leguminous sprouts on genotoxicity, in vitro and in vivo. Cancer Epidemiol. Biomarkers Prev. 13:1199-1205.

Graefe, E.U. and M. Veit. 1999. Urinary metabolites of flavonoids and hydroxycinnamic acids in humans after application of a crude extract from Equisetum arvense. Phytomedicine 6:239-246.

Harper, J.L. 1977. Population biology of plants. Academic Press, New York.

Keles, Y. and I. Öncel. 2002. Response of antioxidative defence system to temperature and water stress combinations in wheat seedlings. Plant Sci. 163:783-790.

Kim, Y., D.W. Giraud, and J.A. Driskell. 2007. Tocopherol and carotenoid contents of selected Korean fruits and vegetables. J. Food Composition Anal. 20:458-465.

Liu, R., S. Xu, J. Li, Y. Hu, and Z. Lin. 2006. Expression profile of a PAL gene from Astragalus membranaceus var. Mongholicus and its crucial role in flux into flavonoid biosynthesis. Plant Cell Rep. 25:705-710.
Llorach, R., F.A. Tomás-Barberán, and F. Ferreres. 2004. Lettuce and chicory byproducts as a source of antioxidant phenolic extracts. J. Agr. Food Chem. 52:5109-5116.

Miller, N.J. and C.A. Rice-Evans. 1996. Spectrophotometric determination of antioxidant activity. Redox Rpt. 2:161-171.

Munné-Bosch, S., K. Schwarz, and L. Alegre. 2001. Water deficit in combination with high solar radiation leads to midday depression of $\alpha$-tocopherol in field-grown lavender (Lavandula stoechas) plants. Aust. J. Plant Physiol. 28:315-321.

Nicolle, C., A. Carnat, D. Fraisse, J. Lamaison, E. Rock, H. Michel, P. Amouroux, and C. Remesy. 2004. Characterisation and variation of antioxidant micronutrients in lettuce (Lactuca sativa folium). J. Sci. Food Agr. 84:2061-2069.

Oh, M.-M. and C.B. Rajashekar. 2009. Antioxidant content of edible sprouts: Effects of environmental shocks. J. Sci. Food Agr. 89:22212227.

Oh, M.-M., E.E. Carey, and C.B. Rajashekar. 2009. Environmental stresses induce health-promoting phytochemicals in lettuce. Plant Physiol. Biochem. 47:578-583.

Olthof, M.R., P.C.H. Hollman, and M.B. Katan. 2001. Chlorogenic acid and caffeic acid are absorbed in humans. J. Nutr. 131:66-71.

Pennycooke, J.C., S. Cox, and C. Stushnoff. 2005. Relationship of cold acclimation, total phenolic content and antioxidant capacity with chilling tolerance in petunia (Petunia $\times$ hybrida). Environ. Exp. Bot. 53:225-232.

Sofo, A., A.C. Tuzio, B. Dichio, and C. Xiloyannis. 2005. Influence of water deficit and rewatering on the components of the ascorbateglutathione cycle in four interspecific Prunus hybrids. Plant Sci. 169:403-412.

Tattini, M., C. Galardi, P. Pìnelli, R. Massai, D. Remorini, and G. Agati. 2004. Differential accumulation of flavonoids and hydroxycinnamates in leaves of Ligustrum vulgare under excess light and drought stress. New Phytol. 163:547-561.

Wimalasiri, P. and R.B.H. Wills. 1983. Simultaneous analysis of ascorbic acid and dehydroascorbic acid in fruit and vegetables by high-performance liquid chromatography. J. Chromatography 256:368-371.

Zobayed, S.M.A., F. Afreen, and T. Kozai. 2007. Phytochemical and physiological changes in the leaves of St. John's wort plants under a water stress condition. Environ. Exp. Bot. 59:109-116. 